\title{
STATUS OF THE FERMILAB ELECTRON COOLING PROJECT
}

\author{
S. Nagaitsev", A. Burov, A.C. Crawford, T. Kroc, J. MacLachlan, \\ C.W. Schmidt, A. Shemyakin ${ }^{+}$, and A. Warner, FNAL", Batavia, IL
}

\begin{abstract}
The first stage of the Fermilab Electron Cooling R\&D program is now complete: technology necessary to generate hundreds of milliamps of electron beam current at $\mathrm{MeV}$ energies has been demonstrated. Conceptual design studies show that with an electron beam current of $200 \mathrm{~mA}$ and with a cooling section of $20 \mathrm{~m}$ electron cooling in the Fermilab Recycler ring can provide antiproton stacking rates suitable for the Tevatron upgrades beyond Run II luminosity goals. A prototype of such an electron cooling system is now being built at Fermilab as part of the continuing R\&D program. This paper describes the electron cooling system design as well as the status of the Fermilab electron cooling $R \& D$ program.
\end{abstract}

\section{INTRODUCTION}

In 1995 Fermilab started an R\&D program in electron cooling that has two principal objectives: (1) to determine the feasibility of electron cooling the $8.9 \mathrm{GeV} / \mathrm{c}$ momentum antiprotons; and (2) to develop and demonstrate the necessary technology. The ultimate goal is to realize a luminosity of $10^{33} \mathrm{~cm}^{-2} \mathrm{~s}^{-1}$ in the Tevatron collider by supplying a larger flux of antiprotons. The conceptual design studies [1] demonstrate that this can be accomplished by providing longitudinal emittance decrements in the Recycler of $200 \mathrm{eVs} / \mathrm{h}$ or higher. The primary technical problem is to generate a high-quality, monochromatic, dc, multi-MeV electron beam of $200 \mathrm{~mA}$ or greater. The technical goal set for a proof-of-principal demonstration using mostly existing equipment was to maintain a $200 \mathrm{~mA}$ beam for a period of one hour. The only technically feasible way to attain such high electron currents is through beam recirculation (energy recovery)[2]. This goal was achieved in 1998 by recirculating beam currents of $200 \mathrm{~mA}$ for periods of up to five hours without a single breakdown. Although the recirculation tests used a 1-1.5 $\mathrm{MeV}$ electron beam and the Fermilab electron cooling system requires a $4.3 \mathrm{MeV}$ beam, the demonstration is relevant because the increased energy does not involve fundamental changes in technology. Currently, Fermilab is building a full-scale, 4.3 MeV electron beam facility to complete the

\footnotetext{
\#Email: nsergei@fnal.gov

${ }^{+}$On leave from the Budker INP, Novosibirsk.

* Operated by the Universities Research Association under contract with the U.S. Department of Energy.
}

experimental $R \& D$ program and to prepare installation of the cooler in the Recycler ring.

\section{RECIRCULATION TEST}

These tests were performed using a 2-MV Pelletron ${ }^{\mathrm{TM}}$ accelerator (Van de Graaff type) at National Electrostatics Corporation (NEC), Middleton, Wisconsin. It is the same accelerator as described in Ref. [3] and [4] with shorter acceleration and deceleration tubes, a new electron gun and collector [5], as well as a different beam line. Figure 1 shows the test beamline layout. Table 1 summarizes the important system parameters. This system employs the Pelletron accelerator with a maximum charging current of a few hundred microamps (typically $50-100 \mu \mathrm{A}$ ). The electron beam line consists of a $7.5 \mathrm{~m}$ long channel with discrete focusing elements (lenses and a $180^{\circ}$ bending magnet) flanked by small aperture $(2.54 \mathrm{~cm}$ ID) acceleration and deceleration tubes.

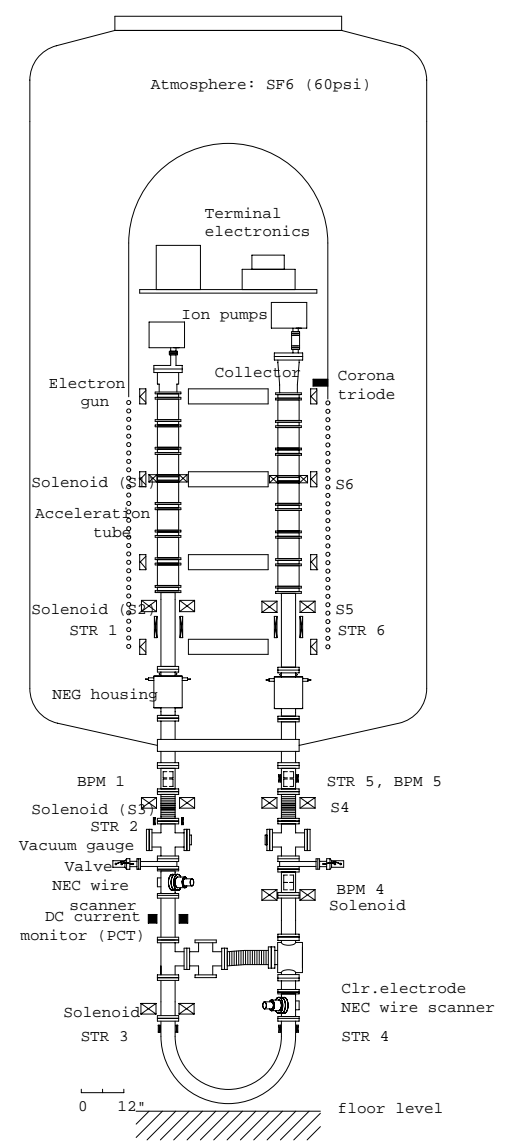

Figure 1: Recirculation system beamline layout. 
Table 1: Recirculation System Parameters

\begin{tabular}{|l|c|c|c|}
\hline \multicolumn{1}{|c|}{ Parameter } & Symbol & Value & Units \\
\hline \hline Pelletron Voltage & $U_{o}$ & $1-1.5$ & $\mathrm{MV}$ \\
Max. Recirculated & & & \\
Beam Current & $I_{b}$ & 0.7 & $\mathrm{~A}$ \\
Typical Vacuum & $p$ & $0.2-1 \times 10^{-7}$ & Torr \\
Relative Losses & $\Delta I / I_{b}$ & $0.5-2 \times 10^{-5}$ & \\
\hline \multicolumn{3}{|c|}{ Electron Gun } \\
\hline Cathode Radius & $R_{c}$ & 1.7 & $\mathrm{~mm}$ \\
Gun Perveance & $P$ & $0.02-0.08$ & $\mu$ Perv \\
Anode Voltage & $U_{A}$ & $\leq 50$ & $\mathrm{kV}$ \\
Control Voltage & $U_{C}$ & $-U_{A} / 13$ & \\
\multicolumn{3}{|c|}{ beam off } \\
beam on
\end{tabular}

The conclusions from the recirculation tests can be summarized as follows:

1. An electron beam with current of hundreds of milliamperes and energy of 1-1.5 $\mathrm{MeV}$ was recirculated in a dc regime. At energy of $1.2 \mathrm{MeV}$ a typical time between crashes is several hours for beam currents of $0.2 \mathrm{~A}$; the maximum recirculated current is $0.7 \mathrm{~A}$. The goal of the test $(0.2 \mathrm{~A}$ during one hour) has been achieved.

2. Relative current losses from the collector do not exceed $5 \times 10^{-6}$ for the beam current of up to $0.5 \mathrm{~A}$.

3. The Pelletron-based recirculation scheme seems to be applicable for the multi-MeV range of beam energy since all of the limitations originate from the low energy part of the system.

\section{ELECTRON BEAM TRANSPORT}

The demonstration of the Pelletron-based electron beam recirculation provides the necessary basis for the next step of the electron cooling R\&D program, electron beam transport. Figure 2 shows the schematic layout of the Recycler electron cooling system. This system is now being reproduced at Fermilab for the full-scale proof-ofprinciple test. Table 2 summarizes the important parameters of this test system. The principal goals for this system are: (1) to demonstrate the recirculation of a 4.3 $\mathrm{MeV}, 0.5$ A electron beam for a period of one hour; (2) to verify that the electron beam quality in the cooling section meets the demands of the electron cooling; and (3) to design the system for the final installation in the Recycler.

Traditional electron cooling devices employ a continuous homogeneous longitudinal magnetic field in the kilogauss range for the beam transport through the cooling region. One of the main reasons is to suppress the transverse velocities arising from the electron beam space charge. In the Recycler system, the space charge effects are much lower because of the higher beam energy. Thus, the longitudinal magnetic field value can be much smaller allowing for a non-traditional transport scheme. Also, the choice of a standard Pelletron accelerator prohibits us from immersing the electron beam line into a continuous magnetic field. Our transport scheme assumes a homogenous longitudinal magnetic field in the gun, collector, and in the cooling section, but a lumped focusing system in between.

Table 1: Electron Cooling System Parameters

\begin{tabular}{|c|c|c|}
\hline Parameter & Value & Units \\
\hline \multicolumn{3}{|c|}{ Electrostatic Accelerator } \\
\hline Terminal Voltage & 4.3 & MV \\
\hline Electron Beam Current & 0.5 & $\mathrm{~A}$ \\
\hline Terminal Voltage Ripple & 500 & V (FWHM) \\
\hline Cathode Radius & 2.5 & $\mathrm{~mm}$ \\
\hline Gun Solenoid Field & $\leq 400$ & $\mathrm{G}$ \\
\hline \multicolumn{3}{|c|}{ Cooling Section } \\
\hline Length & 20 & $\mathrm{~m}$ \\
\hline Solenoid Field & $\leq 100$ & $\mathrm{G}$ \\
\hline Vacuum Pressure & 0.1 & nTorr \\
\hline Electron Beam Radius & 6 & $\mathrm{~mm}$ \\
\hline Electron Beam Divergence & $\leq 80$ & $\mu \mathrm{rad}$ \\
\hline
\end{tabular}

The cooling rates are extremely sensitive to the angles between the electrons and the antiproton beam. Both coherent and incoherent angles must be smaller than $10^{-4}$ radians to avoid cooling degradation. To provide zero angular velocity inside the solenoid, the electrons must enter the solenoid with the correct radius and divergence, determined by the Busch theorem [6].

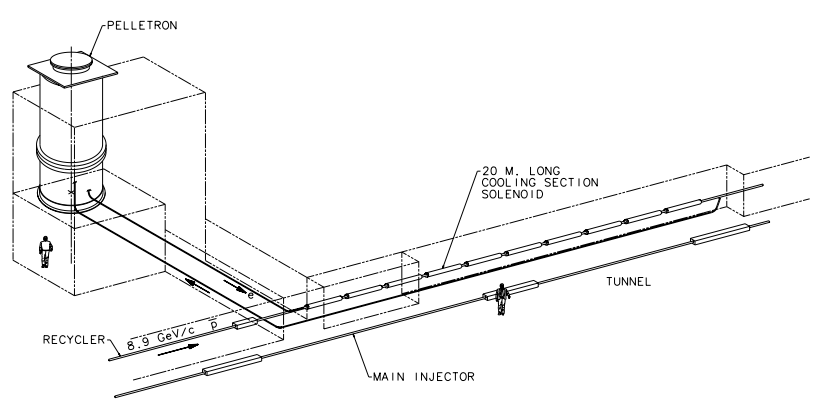

Figure 2: Schematic layout of the Recycler electron system.

The input beam line delivering the electrons to the cooling section has to conserve the rotational invariance. The most natural way to do that is to use axially symmetric optical elements: solenoidal lenses and bending magnets with a field index $n=1 / 2$. At the exit of the input beam line, the beam must be round with the required radius and zero radial angle. The effects of the chromaticity and nonlinearities must be smaller than the angle tolerances. 
The output beam line should also conserve rotational invariance to provide beam collection in the collector without losses.

To simulate the beam transport, a computer code dealing with linear and nonlinear optics was developed. This code was cross-checked for low beam currents with a commercially available beam transport code OptiM 2.1 [7]. The preliminary results for the electron beam envelope using an axially-symmetric beam line are shown in Figure 3. All the requirements are satisfied with this transport line.

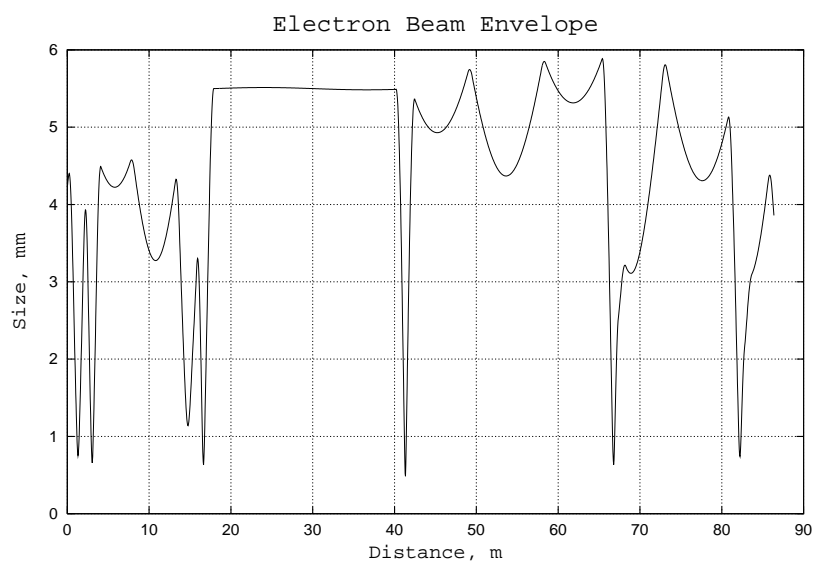

Figure 3: Electron beam envelope.

\section{PROJECT STATUS}

A 5 MV Pelletron accelerator has been ordered and will be installed at Fermilab in July of 2000 in a dedicated building. The initial recirculation tests will proceed with a short beamline similar to the one in Figure 1. Once the recirculation of a $0.5 \mathrm{~A}$ electron beam is demonstrated, the full scale beam line tests will begin. Under the most optimistic scenario the electron cooling system might be installed in the Recycler at the end of FY2001 (contingent upon the collider run schedule).

\section{ACKNOWLEGDEMENTS}

Many ideas and original contributions to this work belong to Anatoly Sharapa who passed away in August of 1998. The authors are thankful to T.N. Andreeva for the assistance in computer simulations and to the entire NEC personnel for their technical support. We would also like to thank V.A. Lebedev for his contribution in beam line modeling, V.V. Parkhomchuk for useful discussions, F. Saffrahn for his help, and G. Saewert for his work on many electronics components. The recirculation experiment was supported in part by the Indiana University Cyclotron Facility through their generous contribution of hardware. The electron gun and collector were manufactured at Budker INP (Novosibrsk) within the framework of the Fermilab - INP collaboration.

\section{REFERENCES}

[1] A. Burov, et al., "Design of Antiproton Electron Cooling in the Recycler", these proceedings.

[2] A.C. Crawford, S. Nagaitsev, A. Sharapa, and A. Shemyakin, "Successful MeV-range electron beam recirculation", in Proc. of EPAC-98 (Stockholm, Sweden, June 22-26, 1998).

[3] L.R. Elias and G. Ramian, IEEE Trans. Nucl. Sci. NS-32 No. 5 (1985), p. 1732.

[4] J.R. Adney, M.L. Sundquist, D.R. Anderson, D.J. Larson, and F.E. Mills, IEEE Proc. of 1989 Particle Accelerator Conf., pp. 348-350 (1989).

[5] A. Sharapa, A. Shemyakin, and S. Nagaitsev, Nucl. Instr. and Meth. A 417 (1998) 177.

[6] S. Nagaitsev et al., "Electron cooling for the Fermilab Recycler Ring", in Proc. of XVII Intl. Conf. On High Energy Accelerators (Dubna, Russia, Sep. 7-12, 1998).

[7] LDBS Co., 110 Bugle Ct., Yorktown, VA 23693 Phone: (757)867-7998, Fax: (757)867-6570 